SHEF-HEP/02-4,

July 2002 ,

Revision No.4

\title{
Inclusive SUSY Searches and Measurements at ATLAS
}

\author{
D.R. Tovey \\ Department of Physics and Astronomy, University of Sheffield, \\ Hounsfield Road, Sheffield S3 $7 R H, U K$.
}

\begin{abstract}
Results of a new study of the discovery potential within mSUGRA parameter space of inclusive searches for SUSY at ATLAS are presented. These results indicate that superior performance is provided by the jets $+E_{T}^{\text {miss }}$ channel in which no requirements are placed upon lepton multiplicity. The sensitivity of this and other channels is mapped in $m_{0}$ $m_{1 / 2}$ parameter space for four different values of $\tan (\beta)$ with similar performance being obtained in all cases. Inclusive measurements of the effective mass scale $M_{\text {susy }}^{\text {eff }}$ and total production cross-section $\sigma_{\text {susy }}$ of supersymmetric particles are also discussed and results presented of a study of the likely measurement precisions.
\end{abstract}

PACS: 12.60.Jv; 14.80.Ly; 04.65.+e

Keywords: LHC physics; supersymmetry; SUGRA; MSSM; inclusive SUSY measurements; jet signature 


\section{General Introduction}

Inclusive searches for SUSY will be one of the most important tasks for ATLAS in the first few years of LHC operation. Although it is not yet clear exactly how such searches will be carried out, they will almost certainly make use of the well-known 'gold-plated' jets $+E_{T}^{\text {miss }}+n$ leptons channel [1]. Many classes of SUSY model predict significant excesses of events in this channel over large areas of parameter space and consequently these searches may provide early evidence for the existence of SUSY. This will not however provide evidence for a specific SUSY model and so techniques must be developed for measuring the properties of an observed signal in a model-independent manner in order to provide input to model-building exercises. The first such measurements are likely to be fits to distributions of inclusive quantities estimating the masses of produced sparticles. These fits will provide information about the SUSY mass scale and inclusive SUSY production cross-section and it is hoped that this will permit the identification of candidate models which can be investigated more fully in subsequent exclusive analyses.

This note describes studies of both classes of inclusive analysis. Following a brief description of the ATLAS detector in Section 2, the results of a study of the discovery potential of inclusive searches are presented in Section 3, with specific reference being made to the benchmark minimal Supergravity (mSUGRA [2]) framework often studied by the LHC community. It should be noted that studies of other classes of SUSY model, including Gauge Mediated SUSY Breaking (GMSB [3]) and Anomaly Mediated SUSY Breaking (AMSB [4]) have also been carried out by ATLAS and are described elsewhere in the literature $[5,6]$. Findings of a study of inclusive mass scale and SUSY production cross-section measurements are presented in Section 4, with an emphasis being placed on model-independence of results.

\section{The ATLAS Detector}

The ATLAS detector is one of two general purpose detectors being constructed for the Large Hadron Collider at CERN. The detector is designed to be sensitive to the full range of high $p_{T}$ physics processes occurring in $14 \mathrm{TeV}$ p-p collisions, with an emphasis on efficient tracking and identification of charged particles, and accurate, large acceptance calorimetric measurement of shower $p_{T}$ and $E_{T}^{\text {miss }}$. The detector consists of the following main components:

- An inner detector for charged particle tracking and identification. Tracking close to the beam-pipe is provided by high granularity, radiation-hard Si microstrip and pixel detectors while at larger radii straw tubes sensitive to transition radiation signals provide both tracking and electron identification. Tracking extends to $|\eta| \sim 2.5$ with full coverage in $\phi$.

- An electromagnetic sampling calorimeter (ECAL) providing electron and photon identification and measurement. The ECAL consists of lead absorber plates immersed in an active liquid argon (LAr) matrix read-out with a maximum granularity of 0.003 in $\eta$ and 0.025 in $\phi$. Electromagnetic calorimetry extends to $|\eta| \sim 3.2$.

- A hadronic sampling calorimeter (HCAL) providing hermetic jet $p_{T}$ and $E_{T}^{\text {miss }}$ measurements over the range $|\eta| \lesssim 5$ with full coverage in $\phi$. In the barrel region $(|\eta|<1.7)$ the HCAL consists of iron absorber plates instrumented with plastic scintillating tiles, 
while in the endcaps $(1.5<|\eta|<3.2)$ LAr modules with $\mathrm{Cu}$ absorbers are used. In the forward region $(3.1<|\eta|<4.9)$ an active LAr matrix is again used, but with a tungsten absorber.

- A stand-alone muon spectrometer for precise muon $p_{T}$ measurement and triggering. Technologies used depend upon pseudorapidity and purpose (tracking or triggering) and include resistive plate chambers, thin-gap chambers, monitored drift tubes and cathode strip chambers. The muon spectrometer is hermetic over the range $|\eta|<2.7$ for all values of $\phi$.

- A magnet system facilitating track $p_{T}$ measurement in the inner detector and muon system consisting of a central 2 Tesla solenoid and three superconducting air-cored toroids spanning the muon spectrometer.

Further details regarding the ATLAS detector and associated trigger and data acquisition systems can be found in [7]. The most important aspects of any detector used for inclusive SUSY studies are the energy resolutions and hermeticities of the calorimeters, since mismeasurement of jets by the ECAL and HCAL can lead to significantly increased rates of high- $E_{T}^{\text {miss }}$ background QCD events. The design of the ATLAS detector in general and the calorimeters in particular has been optimised from the outset so as to minimise these effects.

The studies described in this paper used the ATLFAST [8] fast simulation code to convolve generator-level events with realistic detector energy and position resolutions and particle identification efficiencies. ATLFAST uses a parameterization of the performance of ATLAS sub-detectors which has been developed and verified over the course of several years through comparison with fully simulated and reconstructed data. ATLFAST is therefore believed to model the likely performance of ATLAS with some accuracy.

\section{Inclusive Searches}

\subsection{Introduction}

Monte Carlo studies of inclusive searches for R-Parity conserving SUSY particle production at the LHC have already been carried out by ATLAS $[7,9,10,11]$ and CMS $[12,13]$. These studies have typically worked within the framework of minimal Supergravity (mSUGRA) [2], a constrained subset of the Minimal Supersymmetric Standard Model (MSSM) [14]. While it is probable that mSUGRA models provide only a simple approximation to the rich phenomenology which can emerge from less constrained models, it is believed that mSUGRA is nevertheless a useful benchmark against which detector performance can be assessed. A particularly advantageous feature of mSUGRA models is that the sensitivity of hadron collider experiments to inclusive signatures is governed predominantly by only two parameters, $m_{0}$ and $m_{1 / 2}$ (the GUT scale common scalar and gaugino masses), which determine the masses and production cross-sections of the strongly interacting sparticles (gluinos and squarks).

Following production in LHC collisions, strongly interacting sparticles will decay via a complicated series of cascade processes involving a large number of less massive sfermions, gauginos and Standard Model particles. For R-parity conserving models this will result in high multiplicity events consisting of high $p_{T}$ SM particles and two invisible LSPs (Lightest Supersymmetric Particles). Inclusive searches for SUSY therefore concentrate on channels 
containing jets, $n$ leptons and $E_{T}^{\text {miss }}$, with the required number of leptons being varied depending on the desired signal-to-background ratio and hence sensitivity to systematic uncertainties in background rate [12].

The discovery potentials of specific search channels, defined as contours of constant signal significance, are typically mapped within the $m_{0}-m_{1 / 2}$ parameter space for set values of the other mSUGRA parameters $\left(A_{0}, \tan (\beta)\right.$ and $\left.\operatorname{sign}(\mu)\right)$. The values assumed for these parameters are somewhat arbitrary, however they are usually motivated by the phenomenological evidence available at the time.

\subsection{Event Simulation}

For this study fixed values of $\tan (\beta)$ equal to $10,30,50$ and 55 were chosen in order to span the region of parameter space favoured by LEP data and theory [15]. $\mu$ was taken to be positive throughout as a result of the strong constraints on models with $\mu<0$ provided by $b \rightarrow s \gamma$ measurements $[15,16]$. It is unlikely however that the discovery potential will differ significantly for $\mu<0$ (see for example Fig. 20-3 in Ref. [7]).

In common with previous studies $A_{0}$ was taken to be zero. The results of this inclusive study are however unlikely to be sensitive to the precise value chosen for this parameter due to the weak dependence of the weak scale trilinear couplings $A_{b}$ and $A_{t}$ on the common GUT scale value [7]. The one exception to this is at very large values of $\tan (\beta)$ where the dependence is stronger, possibly leading to increased lepton production and hence enhanced significances in multi-lepton channels $(n \geq 2)$. For such scenarios further $m_{0}-m_{1 / 2}$ scans with non-zero values of $A_{0}$ would be of benefit.

Events were generated using ISA JET 7.51 [17] (SUSY signal) or PYTHIA 6.152 [18] (SM background). The signal events were generated from mSUGRA models $\left(10^{4}\right.$ events/model) lying on a square lattice of spacing $100 \mathrm{GeV}$ in the $m_{0}-m_{1 / 2}$ plane. For the background sample $1 \times 10^{6} \mathrm{t} \overline{\mathrm{t}}, \mathrm{W}+$ jet, $\mathrm{Z}+$ jet and light quark $($ i.e. not $\mathrm{t} \overline{\mathrm{t}}) \mathrm{QCD}$ events were generated in each of five logarithmic $p_{T}$ bins running from a lower $p_{T}$ limit of $50 \mathrm{GeV}$. This procedure was designed to maximise statistics in the high $p_{T}$ region, where the majority of signal-like background events are expected to lie. Detector simulation was carried out using ATLFAST 2.10 [8]. All data was assumed to be collected at low luminosity $\left(10^{33} \mathrm{~cm}^{-2} \mathrm{~s}^{-1}\right)$, with no additional smearing due to pile-up being applied. Future work aimed at assessing the discovery potential resulting from high luminosity running $\left(10^{34} \mathrm{~cm}^{-2} \mathrm{~s}^{-1}\right)$ will incorporate pile-up events and use a more sophisticated fast calorimeter simulation based upon shower parameterisation [19].

\subsection{Data Analysis Technique}

A cut-based analysis was applied to the Monte Carlo data samples in order to estimate detector sensitivity to each mSUGRA model. Cuts were optimised separately for each individual point in mSUGRA parameter space and detector sensitivity assessed using three different significance variables:

1. $S_{f}=\frac{S}{\sqrt{B}}$, with $S \geq 10$ ('ATLAS' variable [7]),

2. $S_{f}=\frac{S}{\sqrt{S+B}}$ ('CMS' variable [12]),

3. $S_{f}=\sqrt{S+B}-\sqrt{B}$ ('combined' variable [20]). 
All three variables quantify signal significance in standard deviation units in the gaussian statistical regime. The ATLAS variable is related to the probability that an observed excess of events $S$ above the expected mean number of background events $B$ is caused by an upfluctuation of the background rather than the presence of a true signal. The CMS variable is related to the accuracy with which a number of signal events $S$ can be measured given the presence of a known number of background events $B$. The CMS variable is perhaps less directly applicable to this class of problem however it does offer the advantage of returning a finite value irrespective of the value of $B$ (which may be zero within errors due to finite Monte Carlo statistics) and without the need for additional explicit constraints on the number of signal events (through an implicit requirement that e.g. $S>25$ for $S_{f}=5$ ). The third ('combined') variable takes into account fluctuations in both background only and signal + background distributions. This technique is more conservative than the other two, which generally give similar results.

\subsection{Optimisation of Cuts}

Cuts on the following variables were found to efficiently reject background without significantly compromising signal efficiencies:

1. $E_{T}^{\text {miss }}$ : Missing $E_{T}$ of event.

2. $p_{T(1)}: p_{T}$ of hardest jet.

3. $p_{T(2)}: p_{T}$ of second hardest jet.

4. $\sum_{i}\left|p_{T(i)}\right|$ : Scalar sum of $p_{T}$ of jets in event.

5. $N_{j}$ : Number of jets in event.

6. $S_{T}$ : Transverse sphericity (circularity) of event.

7. $\Delta \phi_{j}$ : Difference in azimuth between hardest jet and $E_{T}^{\text {miss }}$.

8. $p_{T(l)}: p_{T}$ of hardest lepton (if any).

9. $\Delta \phi_{l}$ : Difference in azimuth between hardest lepton and $E_{T}^{\text {miss }}$.

10. $M_{T}=\sqrt{2 p_{T(l)} E_{T}^{\text {miss }}\left(1-\cos \left(\Delta \phi_{l}\right)\right)}$ : Transverse mass of event (1 lepton channel only).

Jets were found using the standard ATLFAST fixed cone algorithm with a cone size $\Delta R=0.4$ and an uncalibrated $E_{T}$ threshold of $10 \mathrm{GeV}$. Default ATLFAST values were used for all other thresholds and efficiencies. The transverse mass cut (10) was applied only to the one lepton channel in order to reduce the considerable SM background from $\mathrm{W}+$ jet processes. The cut value was fixed at $100 \mathrm{GeV}$ at the beginning of the analysis and not varied thereafter.

Loose pre-selection cuts (Table 1) were applied to all variables in order to reduce the size of the background samples early in the analysis. This had the additional effect of removing regions of cut parameter space near the origin where limited Monte Carlo statistics (particularly for the QCD sample) and deficiencies in the fast simulation package make it impossible to accurately assess the true detector sensitivity. Hard lepton $p_{T}$ cuts corresponding to likely 


\begin{tabular}{||l|l|l|l||}
\hline \hline Cut & Variable & Pre-selection & Upper Limit \\
\hline 1 & $E_{T}^{\text {miss }}$ & $200 \mathrm{GeV}$ & $2000 \mathrm{GeV}$ \\
2 & $p_{T(1)}$ & $100 \mathrm{GeV}$ & $2000 \mathrm{GeV}$ \\
3 & $p_{T(2)}$ & $100 \mathrm{GeV}$ & $2000 \mathrm{GeV}$ \\
4 & $\sum_{i}\left|p_{T(i)}\right|$ & $200 \mathrm{GeV}$ & $2000 \mathrm{GeV}$ \\
5 & $N_{j}$ & 2 & 20 \\
6 & $S_{T}$ & 0.0 & 1.0 \\
7 & $\Delta \phi_{j}$ & 0 & $\pi$ \\
8 & $p_{T(l)}$ & $10 \mathrm{GeV}$ & $2000 \mathrm{GeV}$ \\
9 & $\Delta \phi_{l}$ & 0 & $\pi$ \\
10 & $M_{T}$ & $100 \mathrm{GeV}$ & $100 \mathrm{GeV}$ \\
\hline \hline
\end{tabular}

Table 1: Cuts applied to event selection variables. Pre-selection cuts were applied to all variables with variable harder cuts then applied in order to maximise signal significance. Cuts were permitted to vary between their pre-selection values and the upper limits shown in Column 4.

on-line trigger thresholds (e.g. 20(25) GeV for single isolated muons(electrons) at low luminosity [7]) were not imposed as it is probable that generic jet $E_{T}^{\text {sum }}+E_{T}^{\text {miss }}$ triggers set at $60 \mathrm{GeV}$ $+60 \mathrm{GeV}$ (low luminosity) or $100 \mathrm{GeV}+100 \mathrm{GeV}$ (high luminosity) will be sufficient for this purpose. The use of such hard lepton triggers is in general not required due to the expected dominance of squark and gluino (rather than chargino/neutralino) production processes at the LHC [21]. This is in strong contrast to the situation at the Tevatron where electroweak gaugino channels provide a key discovery signature. At the very highest mass scales direct chargino/neutralino production can contribute significantly to the overall cross-section (see Section 4.6) and in this case alternative search and triggering strategies based upon high $p_{T}$ leptons $+E_{T}^{\text {miss }}$ may prove effective. Consideration of such an approach is however beyond the scope of this paper.

Harder cuts were applied in the next stage of the analysis, with the parameters of all but the transverse mass cut being varied through an iterative procedure in order to optimise the signal significance for each model. The optimisation proceeded in two steps:

1. Cut parameters were varied independently on a coarse lattice in order to find the approximate position of the global maximum in significance. This procedure neglects correlations between cuts but it was found that if the order in which parameters were varied was defined appropriately, then the minimum found by this procedure was often the same as that found by a more time-consuming general search where all parameters were varied independently.

2. Cut parameters were further tuned using the SIMPLEX Monte Carlo method implemented in MINUIT [22]. Although not the recommended MINUIT optimisation algorithm it was found that SIMPLEX was most effective in this case due to the discontinuous nature of the significance surface in parameter space arising from the requirement of an integer number of jets. Details of the ranges over which parameters were allowed to vary can be found in Table 1. Typical final cut parameters for one mSUGRA model and several different channels are given in Table 2. 


\begin{tabular}{|c|c|c|c|c|c|c|c|}
\hline$\overline{\mathrm{Cut}}$ & Variable & $\bar{E}_{T}^{\text {miss }}$ & $\overline{01}$ & $\overline{11}$ & $21 \mathrm{OS}$ & $21 \mathrm{SS}$ & $\overline{31}$ \\
\hline 1 & $E_{T}^{\text {miss }}(\mathrm{GeV})>$ & 1010 & 1010 & 920 & 650 & 470 & 290 \\
\hline 2 & $p_{T(1)}(\mathrm{GeV})>$ & 195 & 290 & 290 & 195 & 100 & 195 \\
\hline 3 & $p_{T(2)}(\mathrm{GeV})>$ & 290 & 385 & 290 & 195 & 195 & 100 \\
\hline 4 & $\sum_{i}\left|p_{T(i)}\right|(\mathrm{GeV})>$ & 1550 & 1550 & 1370 & 1190 & 1010 & 920 \\
\hline 5 & $N_{j}>$ & 12 & 9 & 5 & 3 & 3 & 3 \\
\hline 6 & $S_{T}>$ & 0.15 & 0.15 & 0.10 & 0.00 & 0.00 & 0.00 \\
\hline 7 & $\Delta \phi_{j}>$ & 0.00 & 0.00 & 0.79 & 0.63 & 0.79 & 0.31 \\
\hline 8 & $p_{T(l)}(\mathrm{GeV})>$ & $\mathrm{N} / \mathrm{A}$ & $\mathrm{N} / \mathrm{A}$ & 10 & 10 & 10 & 10 \\
\hline 9 & $\Delta \hat{\phi}_{l}>$ & $\mathrm{N} / \mathrm{A}$ & $\mathrm{N} / \mathrm{A}$ & 0.31 & 0.00 & 0.00 & 0.00 \\
\hline 10 & $M_{T}(\mathrm{GeV})>$ & $\mathrm{N} / \mathrm{A}$ & $\mathrm{N} / \mathrm{A}$ & 100 & $\mathrm{~N} / \mathrm{A}$ & $\mathrm{N} / \mathrm{A}$ & $\mathrm{N} / \mathrm{A}$ \\
\hline & $B(\mathrm{QCD})$ & 0.00 & 0.00 & 0.00 & 1.2 & 1.42 & 18.33 \\
\hline & $B(\mathrm{Zj})$ & 0.03 & 0.14 & 0.19 & 0.44 & 0.33 & 1.01 \\
\hline & $B(\mathrm{Wj})$ & 0.06 & 0.19 & 0.19 & 0.99 & 4.42 & 3.59 \\
\hline & $B(\mathrm{t} \overline{\mathrm{t}})$ & 0.01 & 0.01 & 0.05 & 2.86 & 11.55 & 44.12 \\
\hline & $B$ (total) & 0.10 & 0.34 & 0.43 & 5.49 & 17.72 & 67.05 \\
\hline & $S$ & 10.57 & 13.23 & 12.30 & 10.17 & 10.04 & 10.04 \\
\hline & $S / B$ & 116.7 & 38.91 & 28.71 & 1.85 & 0.57 & 0.15 \\
\hline & $S / \sqrt{B}$ & 35.12 & 22.69 & 18.79 & 4.34 & 2.39 & 1.23 \\
\hline
\end{tabular}

Table 2: Optimised cut values and significances for six different search channels at point $m_{0}$ $=800 \mathrm{GeV}, m_{1 / 2}=800 \mathrm{GeV}, \tan (\beta)=30, A_{0}=0, \mu>0$ in mSUGRA parameter space. ' $E_{T}^{\mathrm{miss}}$ ' refers to the jets $+E_{T}^{\mathrm{miss}}$ channel with no lepton requirement, 'Ol' refers to the lepton veto channel, '1l' the 1 lepton channel, ' $2 l$ OS' the opposite sign dilepton channel, '2l SS' the same sign dilepton channel and '3l' the trilepton channel. Also given for each channel are the number of $Q C D, Z+j e t, W+j e t$ and $\bar{t} \overline{\mathrm{t}}$ background events passing the cuts, the total number of background events passing the cuts, the number of signal events passing the cuts and the signal-to-background ratio. Cuts were optimised according to the requirements of the 'ATLAS' variable and the final row gives the significance according to this definition. 
At this point it is instructive to consider how a cut-based analysis such as that considered here might proceed with real data. Given the high degree of correlation between the events selected by different sets of cuts it is probable that in the absence of a signal low significances $\left(S_{f}<5\right)$ would be found at all probed points in mSUGRA parameter space. Limits could then be set on the SUSY production cross-section at each point using frequentist techniques such as those used at LEP [23] and a limit curve constructed from the ensemble of cross-section bounds and corresponding mSUGRA predictions. If a signal derived from one SUSY model (not necessarily mSUGRA) were present however then it is probable that a high significance would be observed by cuts optimised at several points in mSUGRA space, again due to correlations between the events selected at each point. This high significance region would provide some preliminary information regarding possible parameter values under the mSUGRA assumption, however it is important to note that it would not correspond to a rigorously defined confidence region. The calculation of confidence regions using cuts varying over parameter space is a non-trivial problem and it is likely that alternative estimation techniques would be used in practice.

\subsection{Results}

Maps of discovery potential corresponding to a $5 \sigma$ excess above background $\left(S_{f}=5\right)$ in mSUGRA $m_{0}-m_{1 / 2}$ parameter space using the 'ATLAS' significance variable for $\tan (\beta)=$ 10, 30, 50 and 55 and $10 \mathrm{fb}^{-1}$ integrated luminosity can be found in Fig. 1. The discovery potential for $\tan (\beta)=10$ using the 'CMS' and 'combined' variables is shown in Fig. 2 for comparison. The relative performance of the six channels considered $\left(E_{T}^{\text {miss }}, 01,11,21\right.$ OS, 21 SS and 31) is found to be similar in all cases, irrespective of the chosen significance variable. The $E_{T}^{\text {miss }}$ channel (no lepton requirement) is found to give the greatest discovery potential, covering $\tilde{\mathrm{q}}$ and $\tilde{\mathrm{g}}$ masses $\lesssim 2 \mathrm{TeV}$. The next greatest discovery potential is provided by the lepton veto channel ('0l'), which performs better in this study relative to the $n>0$ lepton channels than in the Detector and Physics Performance TDR [7], possibly due to the use of a more flexible set of cuts. The performance is similar to that found in CMS studies [12]. The performance of the 1 lepton ('11'), opposite sign dilepton ('2l OS'), same sign dilepton ('2l SS') and trilepton channels is similar to that found previously. The absolute discovery potential for each channel is found to be similar for the 'ATLAS' and 'CMS' significance variables but less great for the 'combined' variable, as expected. 


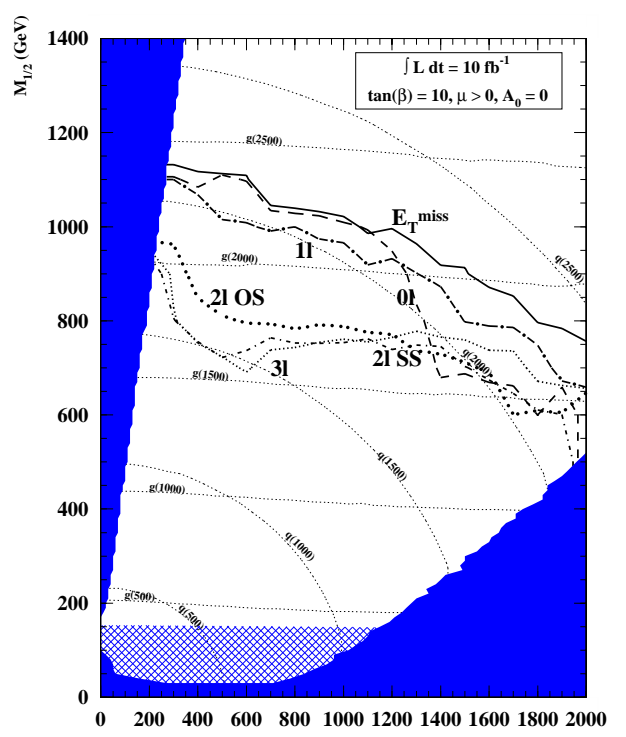

$\mathrm{M}_{0}(\mathrm{GeV})$

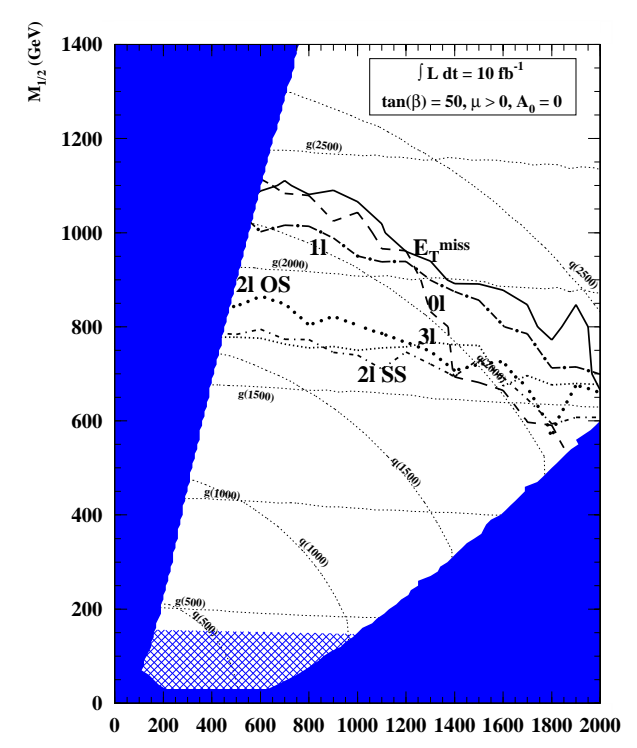

$\mathrm{M}_{0}(\mathrm{GeV})$

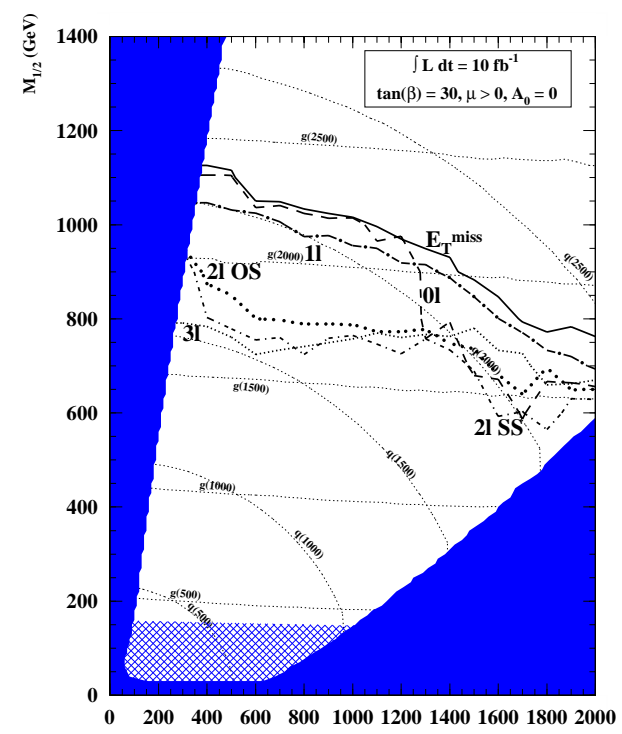

$\mathrm{M}_{0}(\mathrm{GeV})$

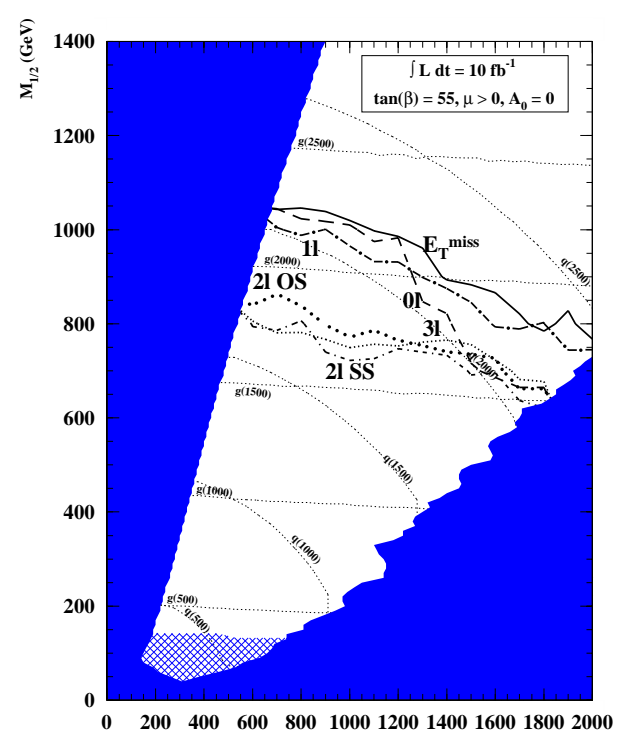

$\mathrm{M}_{0}(\mathrm{GeV})$

Figure 1: $5 \sigma$ discovery potential ('ATLAS' variable) in the $m_{0}-m_{1 / 2}$ plane for $m S U G R A$ models with $\tan (\beta)=10,30,50$ and $55, \mu>0$ and $A_{0}=0$ assuming $10 \mathrm{fb}^{-1}$ integrated luminosity. Bold curves correspond to the $E_{T}^{\mathrm{miss}}$ channel (full curve), ol channel (dashed curve), 11 channel (dash-dotted curve), $2 l$ OS channel (dotted curve), 21 SS channel (dashdash-dotted curve) and $3 l$ channel (small dots). Light curves correspond to squark and gluino iso-mass contours (masses in GeV). ISAJET failed to converge in the full dark regions, while the hatched dark regions are excluded by current experimental bounds from LEP (e.g.Ref. [24]), the Tevatron (e.g.Ref. [25, 26]) and elsewhere. For a recent review of experimental bounds see e.g. Ref. [16] and references contained therein). 


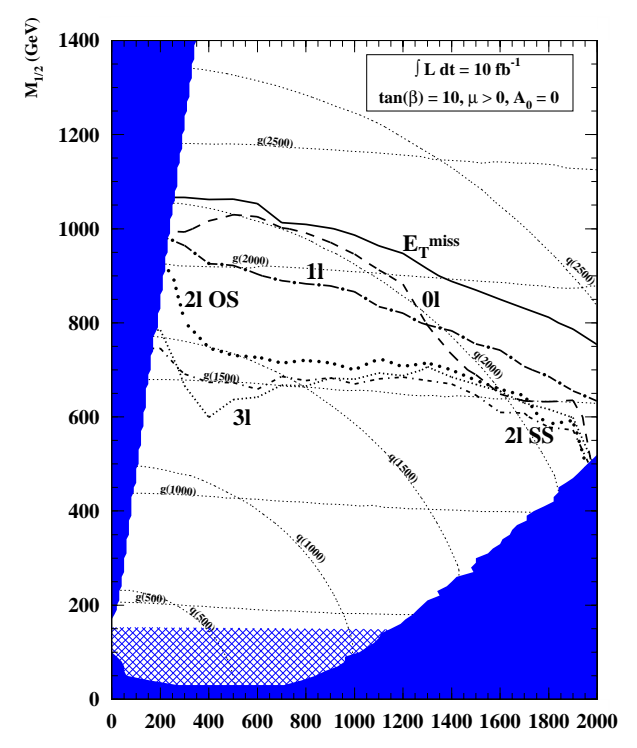

$\mathrm{M}_{0}(\mathrm{GeV})$

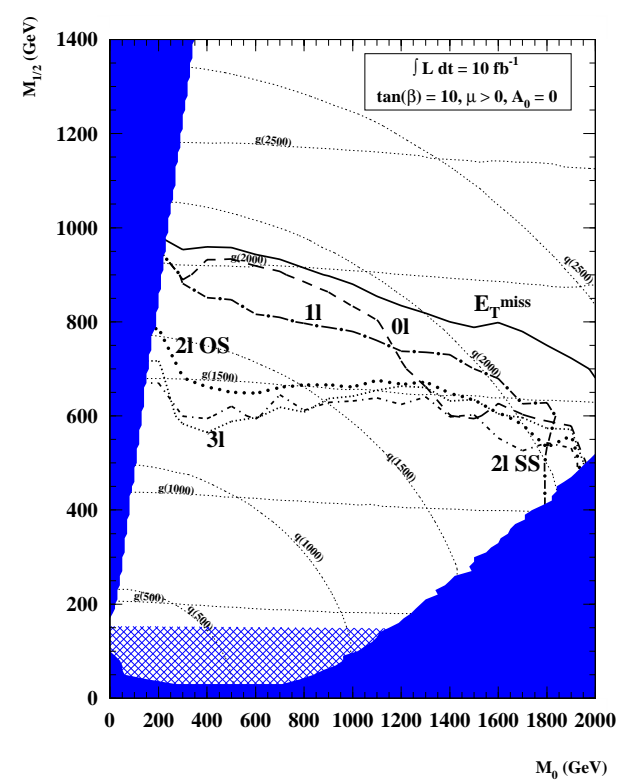

Figure 2: $5 \sigma$ discovery potential in the $m_{0}-m_{1 / 2}$ plane for $m S U G R A$ models with $\tan (\beta)=$ $10, \mu>0$ and $A_{0}=0$ assuming $10 \mathrm{fb}^{-1}$ integrated luminosity. The left-hand panel shows the discovery potential obtained using the 'CMS' significance variable and the right-hand panel the discovery potential obtained with the 'combined' variable (see text). Curves and shaded regions are as for Fig. 1.

In order to assess the effect of systematic errors in estimates of the numbers of SM background events the discovery potential for $\tan (\beta)=10$ of the $E_{T}^{\text {miss }}$ channel ('ATLAS' significance variable) was calculated assuming a factor two increase or decrease in total background cross-section. The results are shown in Fig. 3 and indicate that even a large systematic variation in background rate has only a comparatively small effect on the overall discovery potential.

The discovery potential of ATLAS $(\tan (\beta)=10)$ for specific integrated luminosity during low luminosity running (i.e.assuming no pile-up) is presented in Fig. 4. The discovery potential for even $100 \mathrm{pb}^{-1}$ (< one week of running) covers a significant fraction of the cosmologically and experimentally favoured region of parameter space [15]. In practice however it is unlikely that a discovery could be made in such a short time due to detector calibration and background estimation requirements. In particular, it is probable that the most effective method for estimating SM background contributions will be to use tagged samples of low $E_{T}^{\text {miss }}$ events and then extrapolate into the high $E_{T}^{\text {miss }}$ SUSY signal region. This technique was used by CDF [25] and D0 [26] to estimate the QCD background to the jets $+E_{T}^{\text {miss }}+0$ leptons channel and it is possible that it could be extended to the $\mathrm{Z}+$ jet and $\mathrm{W}+$ jet channels using a tagged sample of $\mathrm{Z} \rightarrow$ ee or $\mu \mu$ events. Estimation of $\mathrm{t} \overline{\mathrm{t}}$ background using similar techniques is more problematic and may require a more conventional approach using large-scale Monte Carlo simulation. Whatever approach is used however it is likely that significant amounts of data will be required before the background contribution can be assessed accurately. 


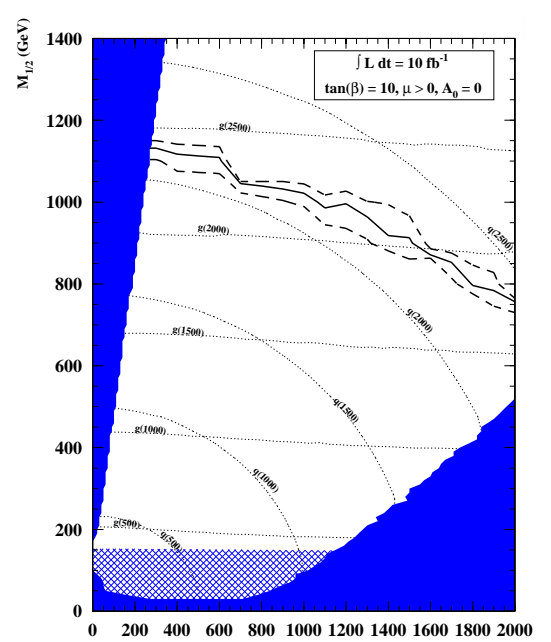

$M_{0}(\mathrm{GeV})$

Figure 3: $5 \sigma$ discovery potential ('ATLAS' variable) of the $E_{T}^{\text {miss }}$ channel in the $m_{0}-m_{1 / 2}$ plane for mSUGRA models with $\tan (\beta)=10, \mu>0$ and $A_{0}=0$ assuming $10 \mathrm{fb}^{-1}$ integrated luminosity and variable SM background cross-section. The full curve corresponds to the discovery potential plotted in Fig. 1 while the two dashed curves correspond to the discovery potential obtained when assuming a factor two increase (lower) or decrease (upper) in SM background cross-section. Shaded regions are as for Fig. 1.

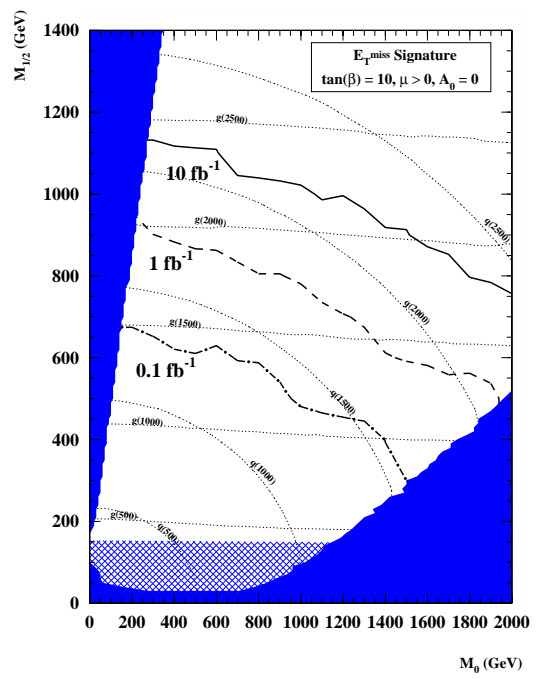

Figure 4: $5 \sigma$ discovery potential ('ATLAS' variable) of the $E_{T}^{\text {miss }}$ channel in the $m_{0}-m_{1 / 2}$ plane for mSUGRA models with $\tan (\beta)=10, \mu>0$ and $A_{0}=0$ assuming $0.1 \mathrm{fb}^{-1}, 1 \mathrm{fb}^{-1}$ and $10 \mathrm{fb}^{-1}$ integrated luminosity. Curves and shaded regions are as for Fig. 1. 


\section{Inclusive Mass Scale Measurements}

\subsection{Introduction}

Techniques for measuring the SUSY mass scale using fits to distributions of inclusive variables derived from the jets $+E_{T}^{\text {miss }}+0$ leptons channel have been described in Refs. [27, 28, 29]. In this section we present the results of a new assessment of one of these techniques using a more accurate parameterization of the ATLAS detector contained in the ATLFAST [8] simulation. Detailed discussion of the variables and technique used can also be found in Ref. [29].

\subsection{Measurement Technique}

The SUSY mass scale at the LHC ( $\left.M_{\text {susy }}\right)$ can be defined as the cross-section weighted mean of the masses of the two (in R-parity conserving models) SUSY particles initially produced in $14 \mathrm{TeV}$ p-p collisions. At low mass scales these particles will predominantly be the lighter squarks and gluinos, although at higher scales there may be a significant contribution from electroweak gauginos due to phase-space suppression of the strongly interacting sparticle production cross-sections. When attempting to measure $M_{\text {susy }}$, the presence of a high mass Lightest Supersymmetric Particle can bias the results by reducing the number and $p_{T}$ of observed jets. In practice it is therefore preferable to consider measurements of an appropriately defined effective mass scale $M_{\text {susy }}^{\text {eff }}$ which takes the LSP mass into account. In general these considerations lead to a complex relationship between $M_{\text {susy }}^{\text {eff }}$ and the masses of produced sparticles but nevertheless any measurement of $M_{\text {susy }}^{\text {eff }}$ can be related to the predictions of a given SUSY model through the use of RGE and coupling formulae such as those implemented in ISASUSY [17].

In Ref. [29] it was found that an accurate variable $M_{\text {est }}$ for measuring $M_{\text {susy }}^{\text {eff }}$ is provided by the scalar sum of the $p_{T}$ of all jets found in an event together with the event $E_{T}^{\text {miss: }}$

$$
M_{\mathrm{est}}=\sum_{i}\left|p_{T(i)}\right|+E_{T}^{\mathrm{miss}},
$$

with an accompanying definition of $M_{\text {susy }}^{\text {eff }}$ in terms of $M_{\text {susy }}$ and the mass of the LSP, $M_{\chi}$ :

$$
M_{\text {susy }}^{\text {eff }}=\left(M_{\text {susy }}-\frac{M_{\chi}^{2}}{M_{\text {susy }}}\right) .
$$

Through the use of appropriate cuts a distribution of $M_{\text {est }}$ values can be constructed in which the SM background population falls monotonically with increasing $M_{\text {est }}$ while the SUSY signal population provides a roughly gaussian peak at a value of $M_{\text {est }}$ proportional to $M_{\text {susy }}^{\text {eff }}$ (Fig. 5). A gaussian fit to the background subtracted $M_{\text {est }}$ distribution can be used to estimate $M_{\text {susy }}^{\text {eff }}$ in a model-independent manner to a high degree of accuracy. As a by-product of this process the fitted normalisation of the signal distribution is found to provide a measure of the total SUSY production cross-section $\sigma_{\text {susy }}$, albeit with more model-dependency. By combining estimates of $M_{\text {susy }}^{\text {eff }}$ and $\sigma_{\text {susy }}$ obtained in this way certain classes of SUSY model may be constrained due to the localisation of model points along specific loci in $M_{\mathrm{susy}}^{\mathrm{eff}}-\sigma_{\text {susy }}$ parameter space. Such behaviour is evident in Fig. 6, where a measurement of $M_{\text {susy }}^{\text {eff }}$ and $\sigma_{\text {susy }}$ lying for instance along the mSUGRA locus would disfavour GMSB [3] models. 


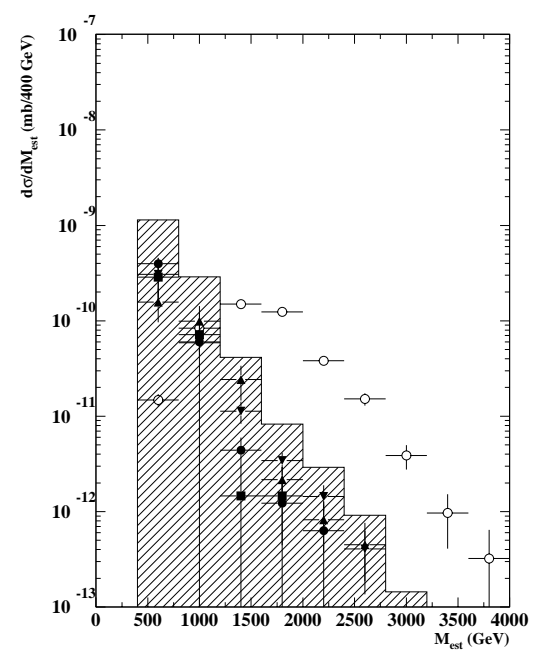

Figure 5: $M_{\mathrm{est}}$ distributions for SM background channels and SUSY signal at ATLAS $m S U G R A$ point $5\left(m_{0}=100 \mathrm{GeV}, m_{1 / 2}=300 \mathrm{GeV}, A_{0}=300 \mathrm{GeV}, \mu>0\right.$ and $\tan (\beta)$ =2.1). SM background contributions are $t \overline{\mathrm{t}}$ (filled circles), $W+$ jet (triangles), $Z+j e t$ (downward triangles) and QCD (filled squares). The hatched histogram corresponds to the sum of all SM backgrounds while the open circles correspond to the SUSY signal population. Error bars indicate statistical errors arising from the finite Monte Carlo sample.

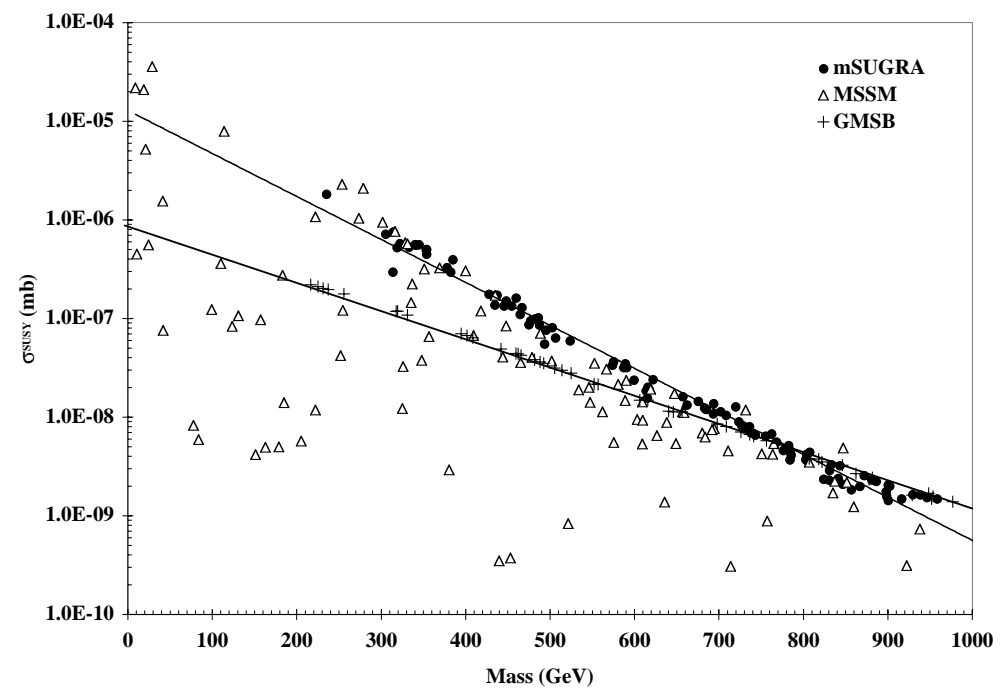

Figure 6: $\sigma_{\text {susy }}$ plotted against $M_{\text {susy }}^{\mathrm{eff}}$ for a collection of mSUGRA models (circles), constrained MSSM models (triangles) and GMSB models (crosses). The mSUGRA and GMSB models lie along the two plotted loci. The constrained MSSM models are scattered more diffusely. 


\subsection{Event Simulation and Selection}

SM background and SUSY signal (mSUGRA and constrained MSSM) events were generated with PYTHIA 6.136 [18]. The constrained MSSM class of models was that implemented in SPYTHIA, incorporating 15 free mass parameters but with no additional D-terms and with 3rd generation trilinear couplings derived from masses. For more details see Ref. [30] and references contained therein. Detector simulation was carried out using ATLFAST 2.10 [8] with a cluster-finding cone size of 0.4 and a jet $E_{T}$ threshold of $50 \mathrm{GeV}$. All other parameters were set to the ATLFAST defaults.

Events in the jets $+E_{T}^{\text {miss }}+0$ leptons channel were selected with the following criteria:

- $\geq 4$ jets with $p_{T} \geq 50 \mathrm{GeV}$

- $\geq 2$ jets with $p_{T} \geq 100 \mathrm{GeV}$

- $E_{T}^{\text {miss }} \geq \max \left(100 \mathrm{GeV}, 0.25 \sum_{i}\left|p_{T(i)}\right|\right)$

- Transverse sphericity (circularity) $S_{T} \geq 0.2$

- No muons or isolated electrons (definition via ATLFAST) in $|\eta|<2.5$.

$5 \times 10^{4} \mathrm{~W}+$ jet, $\mathrm{Z}+$ jet and $\mathrm{t} \overline{\mathrm{t}}$ background events together with $5 \times 10^{5}$ light quark QCD $2 \rightarrow 2$ background events were generated in each of five logarithmic $p_{T}$ bins running from $p_{T}=50 \mathrm{GeV}$. Total ( $p_{T}$ integrated) production cross-sections for background processes were found to be $1.1 \times 10^{-5} \mathrm{mb}$ (W+jet), $4.2 \times 10^{-6} \mathrm{mb}(\mathrm{Z}+$ jet $), 5.3 \times 10^{-7} \mathrm{mb}(\mathrm{t} \overline{\mathrm{t}})$ and $3.0 \times 10^{-2}$ $\mathrm{mb}$ (QCD). The total jet $p_{T}$ threshold of $200 \mathrm{GeV}$ implicit in the event selection was found to prevent bias arising from the $50 \mathrm{GeV}$ hard-process $p_{T}$ threshold used in the generation procedure. On the basis of the statistics generated for each process and the total number of events selected, cuts were estimated to pass less than 1 in $10^{7}$ QCD events and less than 1 in 200 events from the other background channels. Estimation of these rejection efficiencies was however limited by poor statistics (particularly in the QCD case) and so these figures may well be overly optimistic. $1 \times 10^{4}$ SUSY signal events were generated for 100 randomly chosen models in both the mSUGRA and constrained MSSM scenarios.

For mSUGRA models the region of parameter space sampled was specified by the constraints $100 \mathrm{GeV}<m_{0}<500 \mathrm{GeV}, 100 \mathrm{GeV}<m_{1 / 2}<500 \mathrm{GeV},-500 \mathrm{GeV}<A_{0}<500 \mathrm{GeV}$, $1.8<\tan (\beta)<12.0$ and $\operatorname{sign}(\mu)= \pm 1$. The efficiency with which mSUGRA events passed all cuts was found to be $\sim 10 \%$. For constrained MSSM models the masses of the strongly interacting SUSY particles and sleptons were constrained to lie in the range from $250 \mathrm{GeV}$ to $2000 \mathrm{GeV}$ while the mass parameters of the partners of the electroweak gauge bosons were constrained to lie in the range from $50 \mathrm{GeV}$ to the mass of the lightest strongly interacting SUSY particle or slepton. $\tan (\beta)$ was constrained to lie in the range $1.4<\tan (\beta)<100.0$. The cuts used in this study were optimised for large $M_{\text {susy }}^{\text {eff }}$ models to which the Tevatron Run II experiments will be insensitive and consequently only those MSSM models with $M_{\text {susy }}^{\text {eff }}>$ $250 \mathrm{GeV}$ requiring no alteration of cut parameters were retained. Further work could usefully revisit this question in order to examine the degree of complementarity existing between Tevatron and LHC measurements. The efficiency with which constrained MSSM events passed all cuts was again found to be $\sim 10 \%$. 


\subsection{Mass Scale Measurement}

The intrinsic (systematic limited) precision with which $M_{\text {est }}$ can measure $M_{\text {susy }}^{\text {eff }}$ was estimated by fitting gaussian functions to the event distributions for mSUGRA and constrained MSSM models in the absence of background. The fitted means $\overline{M_{\text {est }}}$ of the gaussians were then plotted against the input $M_{\text {susy }}^{\text {eff }}$ values to obtain scatter plots indicating the degree of correlation between the two quantities (Fig. 7). To quantify this degree of correlation the distributions of $\overline{M_{\text {est }}} / M_{\text {susy }}^{\text {eff }}$ values were plotted for mSUGRA and constrained MSSM models having first corrected the data for a non-zero systematic intercept of the data with the $\overline{M_{\text {est }}}(x)$ axis in Fig. 7. This correction was carried out by performing a linear regression on the mSUGRA data (Fig. 7(a)) and then subtracting the fitted intercept from the mSUGRA and constrained MSSM $\overline{M_{\text {est }}}$ values. This procedure approximates that likely to be used with real data, where such an offset would be estimated under the assumption of one particular class of SUSY model through Monte Carlo simulation prior to data analysis. Since the class of SUSY model would be a priori unknown the same offset would be subtracted from data irrespective of whether mSUGRA, constrained MSSM or any other class of model were in fact realised in nature. The distributions of $\overline{M_{\text {est }}} / M_{\text {susy }}^{\text {eff }}$ for mSUGRA and constrained MSSM models obtained in this way are shown in Fig. 8.
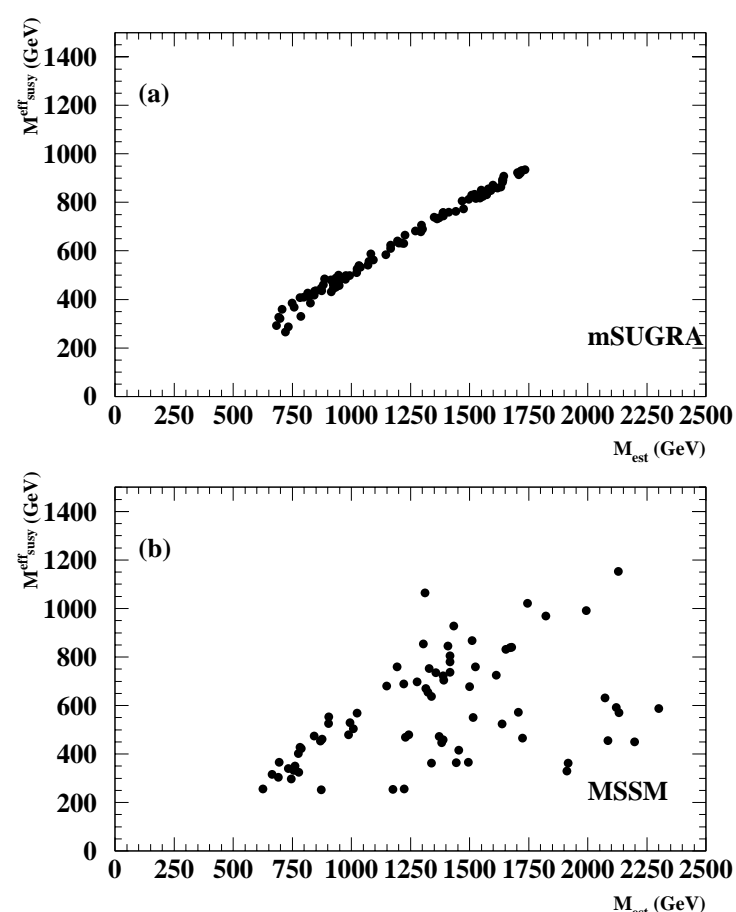

Figure 7: The effective SUSY mass scale $M_{\text {susy }}^{\mathrm{eff}}$ plotted against $\overline{M_{\mathrm{est}}}$ for 100 random mSUGRA models (Fig. $7(a)$ ) and constrained MSSM models (Fig. 7(b)). 

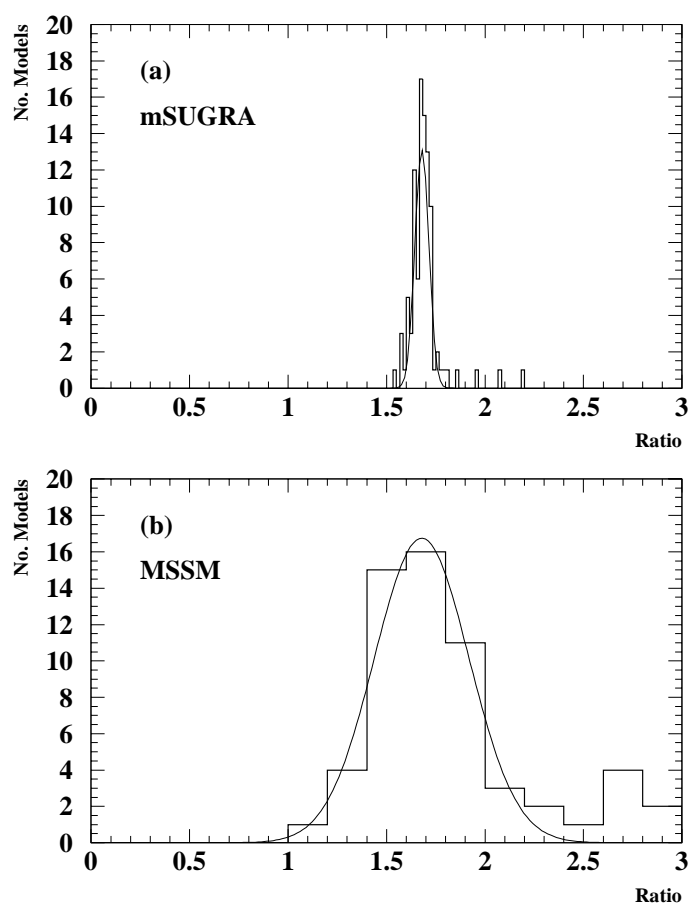

Figure 8: The ratio of $\overline{M_{\mathrm{est}}}$ and $M_{\mathrm{susy}}^{\mathrm{eff}}$ values for data points in Fig. 7 following correction for a non-zero intercept with the $\overline{M_{\text {est }}}$ axis using the technique described in the text. Fig. 8(a) shows the distribution for mSUGRA points and Fig. 8(b) the distribution for constrained MSSM points.

The correlation histograms in Fig. 8 were fitted with gaussian functions in order to obtain estimates of their widths $\sigma$, which measure the intrinsic $M_{\text {susy }}^{\text {eff }}$ measurement precision. Small additional contributions to $\sigma$ arising from statistical scatter in $\overline{M_{\text {est }}}$ values due to finite Monte Carlo statistics were estimated using the rms errors of the $\overline{M_{\text {est }}}$ values for each histogram and subtracted from $\sigma$ in quadrature. Using this technique the intrinsic $M_{\text {susy }}^{\text {eff }}$ measurement precision was estimated to be $2.2 \% \pm 0.3 \%$ for mSUGRA models and $11.1 \% \pm 1.9 \%$ for constrained MSSM models. These results are consistent with those found in Ref. [29]. Note that in addition to the intrinsic precision with which $M_{\text {susy }}^{\text {eff }}$ can be measured using this technique an additional systematic effect due to imperfect calibration of the jet $p_{T}$ and detector $E_{T}^{\text {miss }}$ scales must be taken into account. The systematic uncertainties in these scales are estimated to be $\sim 1 \%$ and $\sim 4 \%$ respectively [7] and using these figures together with the mean relative magnitudes of $\sum_{i}\left|p_{T(i)}\right|$ and $E_{T}^{\text {miss }}$ for selected SUSY signal events an overall contribution to the measurement precision of $\sim 1.35 \%$ was obtained.

Overall $M_{\text {susy }}^{\text {eff }}$ measurement precisions were estimated by taking into account additional statistical and systematic errors arising when estimating $\overline{M_{\text {est }}}$ from realistic (limited statistics signal + background) data samples [29]. Event $M_{\text {est }}$ distributions were constructed from the background and signal samples for each SUSY model considered assuming $10 \mathrm{fb}^{-1}, 100$ 
$\mathrm{fb}^{-1}$ and $300 \mathrm{fb}^{-1}$ integrated luminosity. The mean expected background distribution was then subtracted with an assumed 50\% systematic error arising from lack of knowledge of the shape or normalisation of the true distribution. The background subtracted distributions were fitted with gaussian functions and the $\overline{M_{\text {est }}}$ measurement errors added in quadrature to the intrinsic measurement precisions obtained above and the assumed $1.35 \%$ systematic error due to imperfect calibration of the detector jet and $E_{T}^{\text {miss }}$ energy scales. The resulting overall measurement precisions are plotted in Fig. 9 and indicate that it should be possible to measure $M_{\text {susy }}^{\text {eff }}$ to a precision of $\lesssim 20 \%(\lesssim 60 \%)$ for mSUGRA (constrained MSSM) models after one year of low luminosity running, improving to a precision of $\lesssim 10 \%(\lesssim 30 \%)$ after one year of high luminosity running.
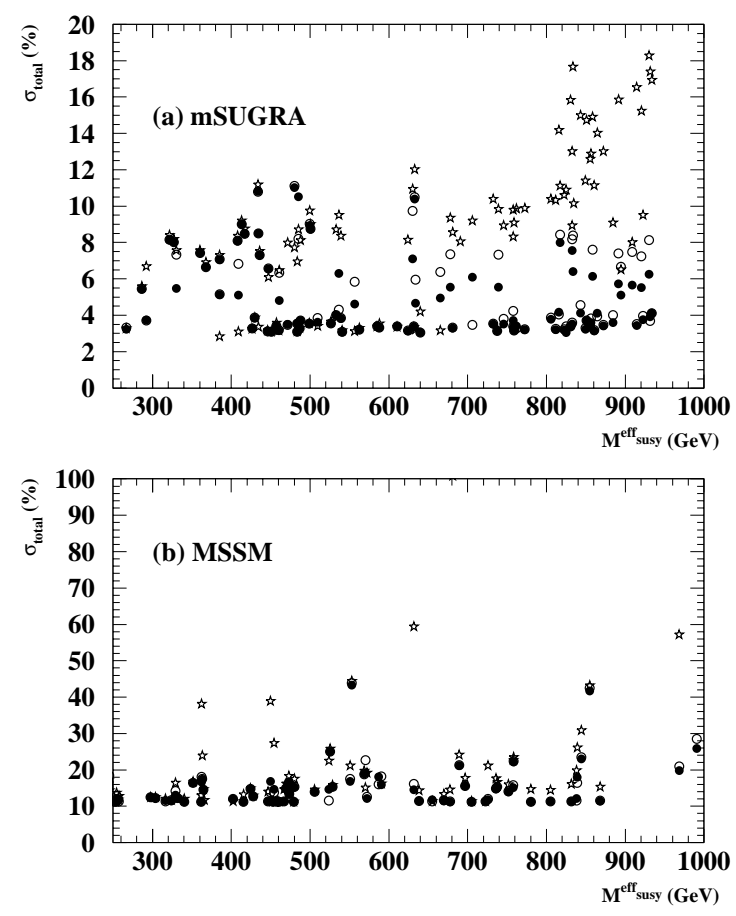

Figure 9: Overall precision for measurement of $M_{\text {susy }}^{\mathrm{eff}}$ after delivery of integrated luminosities of $10 \mathrm{fb}^{-1}$ (stars), $100 \mathrm{fb}^{-1}$ (open circles) and $300 \mathrm{fb}^{-1}$ (filled circles). Precisions for mSUGRA points are plotted in Fig.9(a) and those for constrained MSSM points in Fig. 9(b).

\subsection{Cross Section Measurement}

Measurement of the total SUSY production cross-section $\sigma_{\text {susy }}$ using the fitted normalisation of the distribution of signal event $M_{\text {est }}$ values can be investigated in much the same manner as above. The fitted area under the distribution (hereafter referred to as the 'normalisation' of the distribution and measured in $\mathrm{mb}$ ) is proportional to the total number of SUSY signal events and is hence dependent on $\sigma_{\text {susy }}$. Normalisation- $\sigma_{\text {susy }}$ scatter plots (Fig. 10) indicate 
a high degree of correlation, with points best fitted with a power-law regression curve. This power-law dependency leads to approximately gaussian errors in the natural logarithms of the normalisation and $\sigma_{\text {susy }}$, and consequently it is these quantities which are used to construct the correlation histograms (shown in Fig. 11) following correction of $\ln$ (normalisation) values for a non-zero axis intercept as in Fig. 8. The intrinsic measurement precision for $\ln \left(\sigma_{\text {susy }}\right)$ is found from gaussian fits to these histograms to be $0.9 \% \pm 0.1 \%$ for mSUGRA models and $3.2 \% \pm 0.6 \%$ for constrained MSSM models.
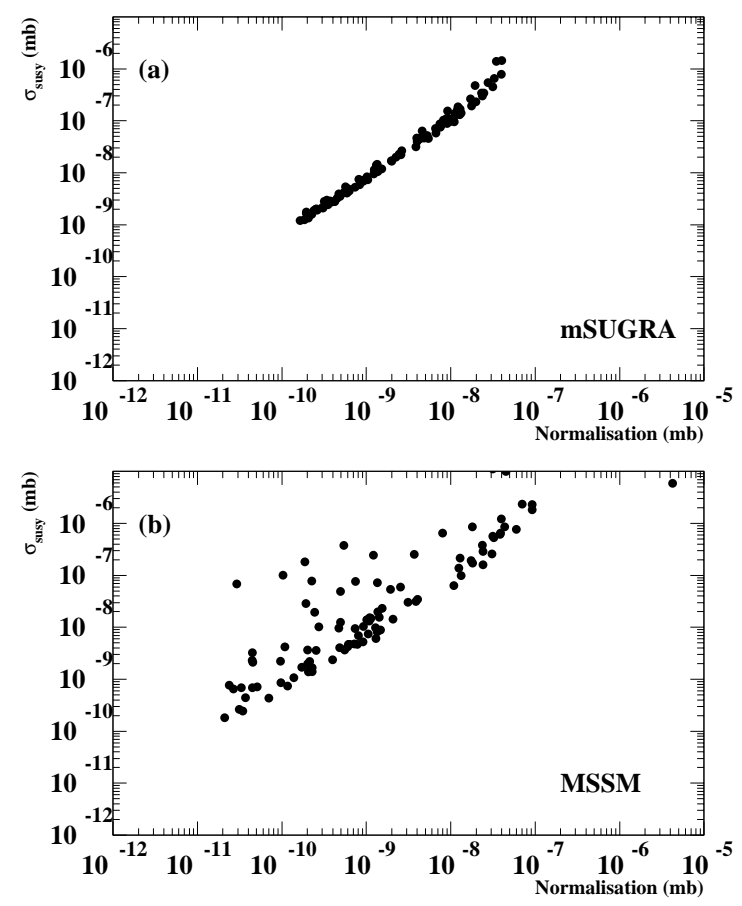

Figure 10: The total SUSY particle production cross section $\sigma_{\text {susy }}$ plotted against the fitted area under the signal distribution (normalisation) for 100 random mSUGRA models (Fig. 10(a)) and constrained MSSM models (Fig. 10(b)). 

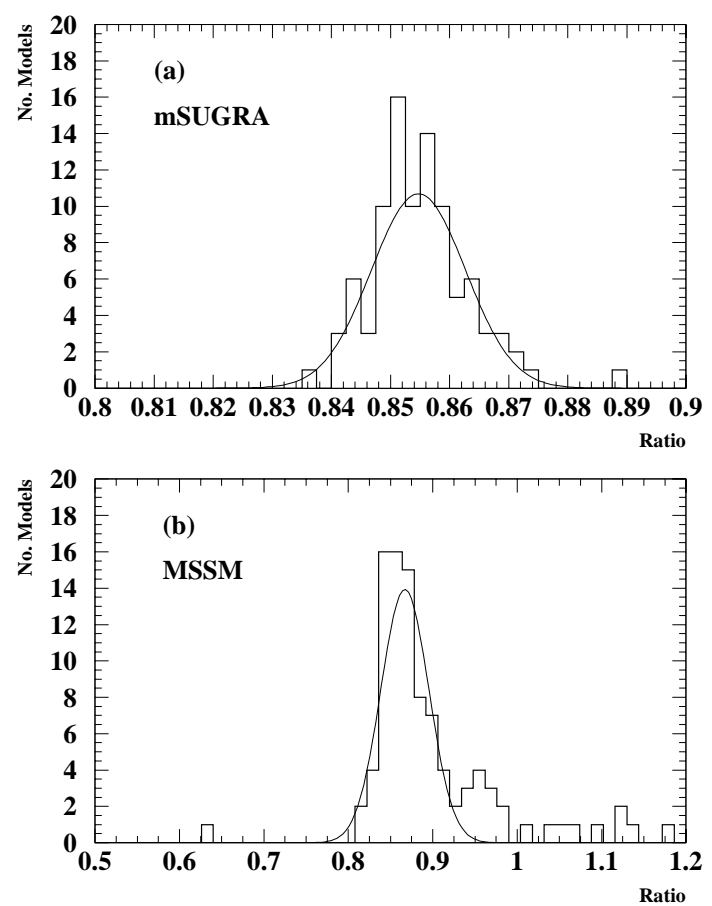

Figure 11: The ratio of natural logarithms of normalisation and $\sigma_{\text {susy }}$ values for data points in Fig. 10 following correction for a non-zero intercept with the normalisation (x) axis using the same technique as was used in Fig. 8. Fig. 11(a) shows the distribution for mSUGRA points and Fig. 11(b) the distribution for constrained MSSM points.

Overall (non-gaussian) measurement precisions for $\sigma_{\text {susy }}\left(i . e . \mathrm{d} \sigma_{\text {susy }} / \sigma_{\text {susy }}=\mathrm{d}\left(\ln \left(\sigma_{\text {susy }}\right)\right)\right)$ were calculated by adding in quadrature the intrinsic measurement precisions and the fitted errors on the normalisations of background subtracted $M_{\text {est }}$ distributions for given integrated luminosity. The results are plotted in Fig. 12 and indicate an ultimate $\left(300 \mathrm{fb}^{-1}\right)$ overall non-gaussian precision of $\lesssim 30 \%$ for mSUGRA models and $\lesssim 80 \%$ for constrained MSSM models. Note that in addition to this non-gaussian precision an additional $5-10 \%$ gaussian systematic error arising from measurement of the integrated luminosity [7] should be taken into account. This contribution is however small when compared with the non-gaussian error. 

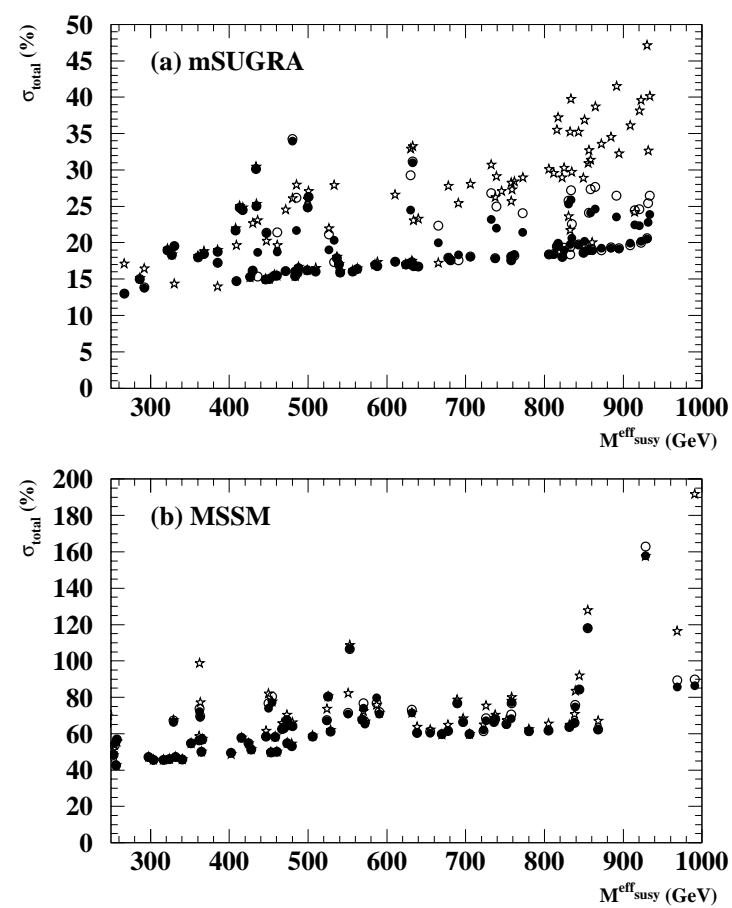

Figure 12: Overall non-gaussian precision for measurement of $\sigma_{\text {susy }}$ after delivery of integrated luminosities of $10 \mathrm{fb}^{-1}$ (stars), $100 \mathrm{fb}^{-1}$ (open circles) and $300 \mathrm{fb}^{-1}$ (filled circles). Precisions for mSUGRA models are plotted in Fig. 12(a) and those for constrained MSSM models in Fig. 12(b).

\subsection{Extension to High Mass Scales}

The studies described here and in Refs. [27, 29] deal with relatively low mass scale SUSY models $\left(m_{\tilde{q}}, m_{\tilde{g}} \lesssim 1000 \mathrm{GeV}\right)$. It is interesting therefore to consider whether this technique can be extended to higher mass scales. In general there is no evidence to suggest that the technique should not work with such models, albeit with reduced measurement precision owing to lack of signal statistics. It is important to note however that in certain regions of model parameter space where both the strongly interacting sparticles and the electroweak gauginos are particularly heavy a new behaviour becomes apparent. The high mass of the strongly interacting sparticles reduces their production cross-sections while that of the lighter electroweak gauginos $\left(\tilde{\chi}_{2}^{0}, \tilde{\chi}_{1}^{ \pm}\right)$causes the corresponding peak in the signal $M_{\text {est }}$ distribution to appear above the selection threshold. As a result of this behaviour the $M_{\text {est }}$ distribution (Fig. 13) can exhibit a double-peaked form, with the lower gaugino peak becoming dominant as the strongly interacting sparticles become progressively heavier. With enough events a gaussian fit to this distribution can still measure $M_{\text {susy }}^{\text {eff }}$ since the result of the fit also tends towards the gaugino mass, however the measurement precision will undoubtedly be reduced due to the inaccuracy of the fitting function. An improved fit using for example a sum of two 
gaussian distributions might be possible in this case and would offer the prospect of obtaining information about both the strongly interacting and weakly interacting sparticle mass scales. It is likely however that the errors obtained from such a fit would be large due to the small number of SUSY signal events (even with $300 \mathrm{fb}^{-1}$ integrated luminosity) and the large number of parameters required to describe the distribution.

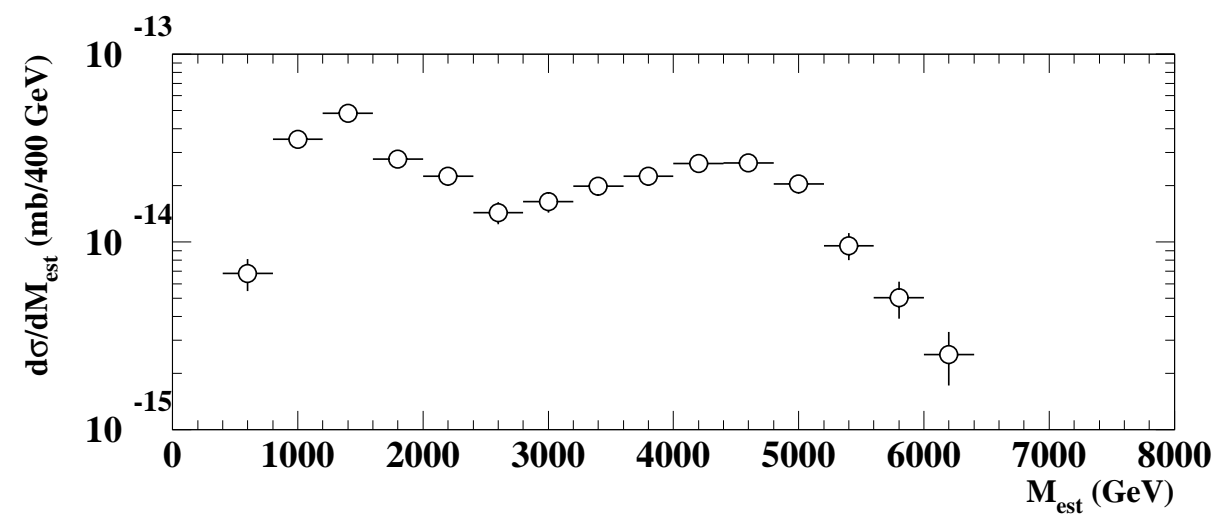

Figure 13: $M_{\mathrm{est}}$ distribution for SUSY signal events generated from a random high mass scale mSUGRA model with $m_{0}=1386 \mathrm{GeV}, m_{1 / 2}=1324 \mathrm{GeV}, A_{0}=-372 \mathrm{GeV}, \tan (\beta)=2$ and $\mu>0$. Inspection of event listings indicates that the right hand peak contains mainly squarks and gluinos while the left hand peak is due predominantly to $\tilde{\chi}_{2}^{0}$ and $\tilde{\chi}_{1}^{ \pm}$production. This model was generated for illustrative purposes only and will not be accessible to ATLAS, even with $300 \mathrm{fb}^{-1}$ integrated luminosity, due to its low cross-section. The model is also already excluded by LEP due to its low value of $\tan (\beta)$.

In mSUGRA parameter space the region where this type of behaviour is dominant lies at large values of $m_{0}$, where the squarks are heavy. This can be seen by constructing a contour plot of $M_{\text {susy }}^{\text {eff }}$ as a function of $m_{0}$ and $m_{1 / 2}$ (Fig. 14). At low $m_{0}, M_{\text {susy }}^{\text {eff }}$ contours run roughly parallel to the $m_{0}$ axis, indicating that the comparatively light squarks dominate the total production cross-section. Above $m_{0} \sim 1000 \mathrm{GeV}$ however the iso-mass scale contours bend upwards indicating that as the squarks become progressively heavier the electroweak gauginos become dominant, with the curves tending towards the gaugino iso-mass contours (see e.g. Fig. 20-2 in Ref. [7]). A measurement of $M_{\text {susy }}^{\text {eff }}$ in this case would favour an elongated strip in $m_{0}-m_{1 / 2}$ parameter space lying between these contours. 


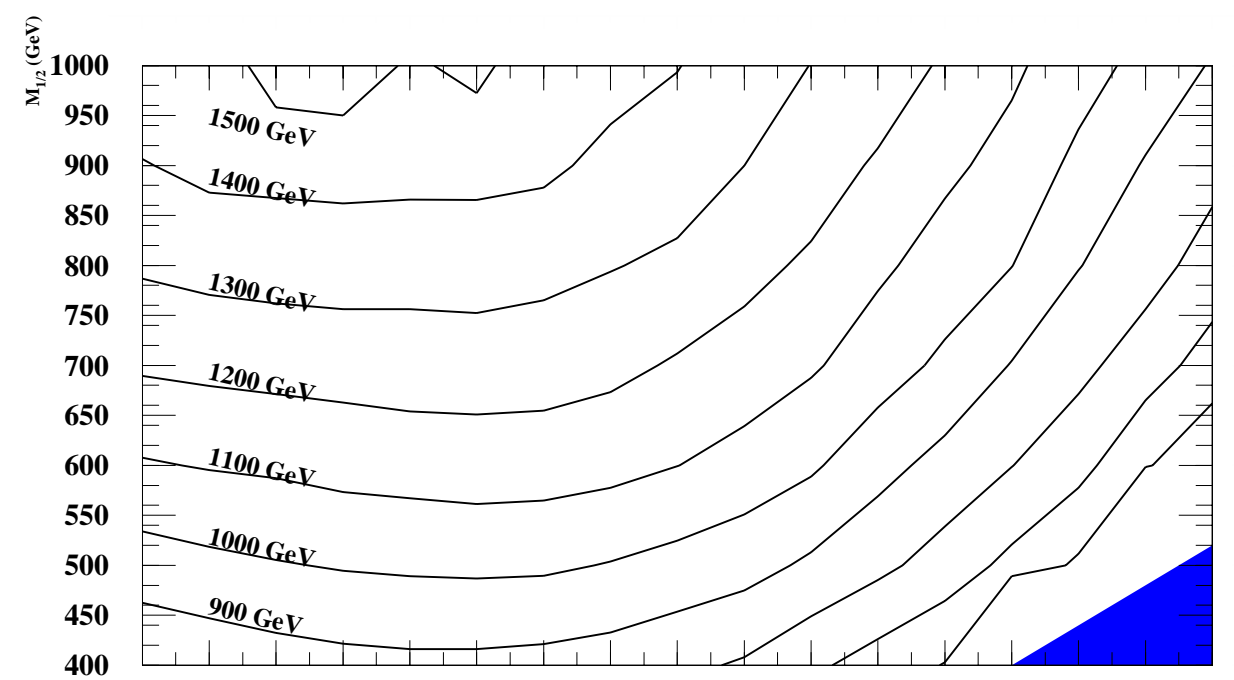

$400500 \quad 60070080090010001100120013001400150016001700180019002000$

$\mathbf{M}_{0}(\mathrm{GeV})$

Figure 14: Contours of constant $M_{\text {susy }}^{\text {eff }}$ in the $m_{0}-m_{1 / 2}$ plane for $m S U G R A$ models with $\tan (\beta)$ $=10, A_{0}=0$ and $\mu>0$. ISA JET failed to converge in the full dark region.

\section{Conclusions}

Studies have been performed of the sensitivity of inclusive SUSY particle searches at ATLAS to mSUGRA parameter space and of inclusive measurements to the effective SUSY mass scale $M_{\text {susy }}^{\text {eff }}$ and production cross-section $\sigma_{\text {susy. }}$. Inclusive searches will be sensitive to models with squark and gluino masses $\lesssim 2 \mathrm{TeV}$ for $10 \mathrm{fb}^{-1}$ integrated luminosity independent of the value of $\tan (\beta)$. The greatest discovery potential is obtained with the inclusive jets + $E_{T}^{\text {miss }}$ channel incorporating no lepton requirements, followed by the zero lepton, one lepton and multi-lepton channels. Inclusive measurements of the effective SUSY mass scale $M_{\text {susy }}^{\text {eff }}$ indicate that precisions $\lesssim 10 \%(30 \%)$ should be obtainable after one year of high luminosity running for mSUGRA (constrained MSSM) models.

\section{Acknowledgments}

The author wishes to thank Frank Paige and Giacomo Polesello for their many helpful comments and suggestions when carrying out this work. The work was performed under the auspices of the SUSY Working Group of the ATLAS Collaboration, making use of a physics simulation framework which is the result of collaboration-wide efforts. The author wishes to acknowledge PPARC for providing financial support. 


\section{References}

[1] H. Baer, X. Tata and J. Woodside, Phys. Rev. D45 (1992) 142.

[2] L. Alvarez-Gaume, J. Polchinski and M.B. Wise, Nucl. Phys. B221 (1983) 495; L. Ibanez, Phys. Lett. B118 (1982) 73; J. Ellis, D.V. Nanopoulos and K. Tamvakis, Phys. Lett. B121 (1983) 123; K. Inoue et al., Prog. Theor. Phys. 68 (1982) 927; A.H. Chamseddine, R. Arnowitt and P. Nath, Phys. Rev. Lett. 49 (1982) 970.

[3] M. Dine, W. Fischler and M. Srednicki, Nucl. Phys. B189 (1981) 575; S. Dimopoulos and S. Raby, Nucl. Phys. B192 (1981) 353.

[4] L. Randall and R. Sundrum, Nucl. Phys. B557 (1999) 79; G.F. Giudice, M.A. Luty, H. Murayama and R. Rattazzi, JHEP 12 (1998) 027.

[5] I. Hinchliffe and F.E. Paige, Phys. Rev. D60 (1999) 095002 (ATLAS NOTE ATL-PHYS98-134).

[6] F.E. Paige and J. Wells, hep-ph/0001249.

[7] ATLAS Collaboration, Detector and Physics Performance Technical Design Report, CERN/LHCC/99-15.

[8] E. Richter-Was, D, Froidevaux and L. Poggioli, ATLAS NOTE ATL-PHYS-98-131.

[9] H. Baer, C.-H. Chen, F. Paige and X. Tata, Phys. Rev. D52 (1995) 2746; Phys. Rev. D53 (1996) 6241.

[10] ATLAS Collaboration, Technical Proposal, CERN/LHCC/94-43.

[11] B.C. Allanach, J.P.J. Hetherington, M.A. Parker and B.R. Webber, JHEP 8 (2000) 17.

[12] S. Abdullin and F. Charles, Nucl. Phys. B547 (1999) 60; M. Dzelalija, Z. Antunovic, S. Abdullin and F. Charles, Mod. Phys. Lett. A15 (2000) 465.

[13] S. Abdullin et al., J. Phys. G28 (2002) 469.

[14] H.P. Nilles, Phys. Rep. 111 (1984) 1; H.E. Haber and G.L. Kane, Phys. Rep. 117 (1985) 75 .

[15] J. Ellis, K.Olive and Y. Santoso hep-ph/0202110.

[16] A. Djouadi, M. Drees and J.L. Kneur, JHEP 0108 (2001) 055.

[17] F. Paige and S. Protopopescu, in Supercollider Physics, ed. D. Soper (World Scientific, 1986); H. Baer, F. Paige, S. Protopopescu and X. Tata, in Proc. Workshop on Physics at Current Accelerators and Supercolliders, ed. J. Hewett, A. White and D. Zeppenfeld (Argonne National Laboratory, 1993).

[18] T. Sjöstrand, Comput. Phys. Commun. 82 (1994) 74.

[19] K. Mahboubi, Private Communication. 
[20] S.I. Bityukov and N.V. Krasnikov, hep-ph/9908492.

[21] F.E. Paige and G. Polesello, Private Communication.

[22] F. James, MINUIT Reference Manual, CERN Program Library Long Writeup D506.

[23] First Workshop on Confidence Limits, ed. F. James, L. Lyons and Y. Perrin, CERN2000-005.

[24] LEPSUSYWG, ALEPH, DELPHI, L3 and OPAL experiments, note LEPSUSYWG/0103

[25] F. Abe et al., Phys. Rev. Lett. 76 (1996) 2006.

[26] B. Abbott et al., Phys. Rev. Lett. 83 (1999) 493724.

[27] I. Hinchliffe, F.E. Paige, M.D. Shapiro, J. Söderqvist and W. Yao, Phys. Rev. D55 (1997) 55209 (ATLAS NOTE ATL-PHYS-97-109).

[28] F.E. Paige, hep-ph/9801254.

[29] D.R. Tovey, Phys. Lett. B498 (2001) 1 (ATLAS NOTE ATL-PHYS-2002-013).

[30] S. Mrenna, Comput. Phys. Commun. 101 (1997) 232. 